\title{
On the Invariant Cantor Sets of Period Doubling Type of Infinitely Renormalizable Area-Preserving Maps
}

\author{
Dan Lilja $『$ \\ Department of Mathematics, Uppsala University, Box 48075106 Uppsala, Sweden. \\ E-mail: dan.lilja@math.uu.se
}

Received: 25 January 2017 / Accepted: 11 September 2017

Published online: 7 November 2017 - (C) The Author(s) 2017. This article is an open access publication

\begin{abstract}
Since its inception in the 1970s at the hands of Feigenbaum and, independently, Coullet and Tresser the study of renormalization operators in dynamics has been very successful at explaining universality phenomena observed in certain families of dynamical systems. The first proof of existence of a hyperbolic fixed point for renormalization of area-preserving maps was given by Eckmann et al. (Mem Am Math Soc 47(289):vi+122, 1984). However, there are still many things that are unknown in this setting, in particular regarding the invariant Cantor sets of infinitely renormalizable maps. In this paper we show that the invariant Cantor set of period doubling type of any infinitely renormalizable area-preserving map in the universality class of the EckmannKoch-Wittwer renormalization fixed point is always contained in a Lipschitz curve but never contained in a smooth curve. This extends previous results by de Carvalho, Lyubich and Martens about strongly dissipative maps of the plane close to unimodal maps to the area-preserving setting. The method used for constructing the Lipschitz curve is very similar to the method used in the dissipative case but proving the nonexistence of smooth curves requires new techniques.
\end{abstract}

\section{Introduction}

The study of renormalization techniques in dynamics began in the 1970s in independent efforts by Feigenbaum [6,7] and Coullet and Tresser [20] to explain the observed universality phenomena in families of maps on the interval undergoing period doubling bifurcation. Acting as a microscope, the renormalization operator can describe the geometric structure of the maps in question at smaller and smaller scales. The existence of a hyperbolic fixed point of the renormalization operator explains the observed universality. It has also been shown that the infinitely renormalizable maps, i.e., those contained in the stable manifold of the renormalization fixed point, have invariant Cantor sets. The dynamics of any two infinitely renormalizable maps restricted to their respective invariant Cantor sets are topologically conjugate. This naturally leads to the question of 
whether or not this conjugacy can be smooth. If there is always a smooth conjugacy we say that the invariant Cantor sets are rigid. It turns out that for infinitely renormalizable unimodal maps of the interval the invariant Cantor sets are indeed rigid.

Since the introduction of renormalization in dynamics this formalism has been generalized in different directions. It has been particularly successful in one-dimensional dynamics, explaining universality for unimodal maps, critical circle maps and maps with a Siegel disk (see e.g. $[1,4,12,15,18,19,21,22]$ and references therein).

The rigorous study of renormalization for dissipative two-dimensional systems was started by Collet et al. [2]. There they define a renormalization operator for strongly dissipative Hénon-like maps and show that the one-dimensional renormalization fixed point is also a hyperbolic fixed point for nearby dissipative maps. This result explains observed universality in families of such maps. In a subsequent paper by Gambaudo et al. [13] it is shown that the infinitely renormalizable maps, i.e., those contained in the stable manifold of the hyperbolic fixed point, have an invariant Cantor set on which the dynamics is conjugate to the dyadic adding machine. A different renormalization operator for strongly dissipative Hénon-like maps was defined by de Carvalho et al. [3]. For this operator they show, in addition to the previous results, that the invariant Cantor sets are not rigid. More precisely, they show that a topological invariant of infinitely renormalizable strongly dissipative Hénon-like maps called the average Jacobian is an obstruction to rigidity. If two infinitely renormalizable maps have different average Jacobians the conjugacy between their respective invariant Cantor sets cannot be smooth. Instead there is a form of probabilistic rigidity. Moreover, they show that there is no smooth curve containing the invariant Cantor set of any infinitely renormalizable map. In Lyubich and Martens [17] also state that every invariant Cantor set of this form is contained in a rectifiable curve. For a proof, see the preprint [16].

In Eckmann et al. [5] introduced a renormalization operator for area-preserving maps of period doubling type of the plane and proved, using computer assistance, the existence of a hyperbolic fixed point. This explains previously observed universality phenomena in families of such maps. Further investigations of this renormalization have been done by Gaidashev and Johnson [8-10] and by Gaidashev et al. [11]. In these papers they prove existence of period doubling invariant Cantor sets for all infinitely renormalizable maps and also show that they are rigid. Again this rigidity can be compared to the situation for dissipative maps where the average Jacobian is a topological invariant and an obstruction to rigidity. Since all area-preserving maps have the same average Jacobian, one would expect, if indeed it is such a classifying invariant, that these Cantor sets are rigid. In [11] a conjecture is made that the average Jacobian is indeed such a classifying invariant.

In the present paper we address two more issues concerning the invariant Cantor sets of infinitely renormalizable area-preserving maps that draw parallels to the situation for dissipative maps. More precisely, we will explore the existence of Lipschitz or smooth curves containing these sets. As in the dissipative case it turns out that there are always Lipschitz curves containing the invariant Cantor sets but there is never a smooth curve.

\subsection{Structure of the paper.}

In Sect. 2, we begin by giving a short introduction to the renormalization of areapreserving maps where we define the necessary objects and state the known results that will be used in the rest of the paper. At the end of Sect. 2 we state the main results of this paper.

Section 3 deals with the existence of a Lipschitz curve containing the invariant Cantor set of each infinitely renormalizable area-preserving map. 
Lastly, in Sect. 4, we prove that there are no smooth curves containing the invariant Cantor set of any infinitely renormalizable area-preserving map.

\section{Area-Preserving Renormalization}

We consider two slightly different renormalization schemes: the one introduced by Eckmann et al. [5] and the one used by Gaidashev et al. [11]. Both schemes are defined for exact symplectic diffeomorphisms of subsets of $\mathbb{R}^{2}$. The maps are also required to be reversible, i.e. $T \circ F \circ T=F^{-1}$ where $T(x, y)=(x,-y)$ for every $(x, y) \in \mathbb{R}^{2}$, and satisfy the twist condition

$$
\frac{\partial X}{\partial y} \neq 0
$$

where $(X, Y)=F(x, y)$. In [11] it is also required that $F(0,0)=(0,0)$. For such maps it is possible to find a generating function $S=S(x, X)$ such that

$$
\left(\begin{array}{c}
x \\
-S_{1}(x, X)
\end{array}\right) \stackrel{F}{\mapsto}\left(\begin{array}{c}
X \\
S_{2}(x, X)
\end{array}\right),
$$

where subscripts denote partial derivatives. It can be shown that $S_{1}(X, x)=S_{2}(x, X) \equiv$ $s(x, X)$ so that $F$ can also be written as

$$
\left(\begin{array}{c}
x \\
-s(X, x)
\end{array}\right) \stackrel{F}{\mapsto}\left(\begin{array}{c}
X \\
s(x, X)
\end{array}\right) .
$$

Using this formulation it is possible to write the differential DF in terms of $s$ using implicit differentiation of $y=-s(X, x)$ and $Y=s(x, X)$. The result is

$$
\mathrm{D} F(x, y)=\left(\begin{array}{cr}
-\frac{s_{2}(X(x, y), x)}{s_{1}(X(x, y), x)} & -\frac{1}{s_{1}(X(x, y), x)} \\
s_{1}(x, X(x, y))-s_{2}(x, X(x, y)) \frac{s_{2}(X(x, y), x)}{s_{1}(X(x, y), x)} & -\frac{s_{2}(x, X(x, y))}{s_{1}(X(x, y), x)}
\end{array}\right) .
$$

Letting $\Lambda[F](x, y)=\left(\lambda_{F} x, \mu_{F} y\right)$ and $\psi_{0}[F](x, y)=\left(\lambda_{F} x+p_{F}, \mu_{F} y\right)$ the two renormalization schemes are then defined by

$$
\begin{aligned}
R_{E K W}(F) & =\Lambda^{-1}[F] \circ F^{2} \circ \Lambda[F] \\
R_{G J M}(F) & =\psi_{0}^{-1}[F] \circ F^{2} \circ \psi_{0}[F]
\end{aligned}
$$

respectively. Here $\lambda_{F}, \mu_{F}, p_{F}$ depend analytically on the map $F$. In the remainder of the paper we will suppress $F$ to avoid notational clutter.

The existence of a hyperbolic fixed point for the EKW renormalization operator was proven in [5] using computer assistance. Similarly the operator $R_{G J M}$ also has a hyperbolic fixed point which is related to the fixed point of $R_{E K W}$ by a translation, as we will see later in the paper. We denote the fixed points by $F_{E K W}$ and $F_{G J M}$ respectively. For the renormalization fixed points the values of the rescalings used to define the renormalization operators are

$$
\begin{aligned}
& \lambda_{E K W}=\lambda_{G J M}=\lambda=-0.249 \ldots, \\
& \mu_{E K W}=\mu_{G J M}=\mu=0.061 \ldots,
\end{aligned}
$$


see e.g. [10]. The Fréchet derivative $\mathcal{D} R_{E K W}$ has two eigenvalues outside the unit circle. One of them corresponds to the universal scaling observed in families of area-preserving maps and the other is $\lambda^{-1}$. The eigenvalue $\lambda^{-1}$ turns out to be related to a translational change of variables and this eigenvalue is eliminated by using the rescalings of $R_{G J M}$. Thus at the fixed point $F_{E K W}$ the renormalization $R_{E K W}$ has a codimension 2 stable manifold whereas at the fixed point $F_{G J M}$ the renormalization $R_{G J M}$ has a codimension 1 stable manifold. We denote the stable and unstable manifolds by $W^{s}(F)$ and $W^{u}(F)$ respectively. Maps $F$ contained in either of their stable manifolds $W^{s}\left(F_{E K W}\right)$ or $W^{s}\left(F_{G J M}\right)$ will be called infinitely renormalizable with respect to the corresponding renormalization operator.

In addition to being defined for area-preserving maps of the plane, $R_{E K W}$ is also defined on the generating functions themselves according to

$$
R_{E K W}(s)(x, X)=\mu^{-1} s(z(x, X), \lambda X)
$$

where $z$ is a symmetric function, $z(x, X)=z(X, x)$, satisfying the equation

$$
s(\lambda x, z(x, X))+s(\lambda X, z(x, X))=0 .
$$

The following normalizations are used for the EKW renormalization in [10] and will be of use to us:

- $s(1,0)=0$,

- $s_{1}(1,0)=1$,

- $z(1,0)=z(0,1)=1$.

- $\mu=z_{1}(1,0)$,

For more details on this see, for example, [5] or [10].

For any $F \in W^{S}\left(F_{G J M}\right)$ there are analytically defined simply connected domains $B_{0}(F)$ and $B_{1}(F)$ that are disjoint and satisfy

- $\quad F^{2}\left(B_{0}(F)\right) \cap B_{0}(F) \neq \emptyset$, and

- $\psi_{w}\left(B_{0}(R F) \cup B_{1}(R F)\right) \subset B_{w}(F)$

where $w \in\{0,1\}$ and $\psi_{1}=F \circ \psi_{0}$. It is then possible to define a nested sequence of sets $B_{w}^{n}$ for $w \in\{0,1\}^{n}$ by

$$
\begin{aligned}
& B_{w 0}^{n}=\psi_{w}^{n}\left(B_{0}\left(R^{n} F\right)\right) \\
& B_{w 1}^{n}=\psi_{w}^{n}\left(B_{1}\left(R^{n} F\right)\right)
\end{aligned}
$$

where $\psi_{w}^{n}=\psi_{w_{1}}[F] \circ \cdots \circ \psi_{w_{n}}\left[R^{n-1} F\right]$ for $w=\left\{w_{1}, \ldots, w_{n}\right\} \in\{0,1\}^{n}$.

The sets $B_{w}^{n}$ are nested as follows:

$$
B_{w v}^{n} \subset B_{w}^{n-1}
$$

for $w \in\{0,1\}^{n}$ and $v \in\{0,1\}$, see Lemma 3.3 of [11]. This gives us the following schematic picture of the renormalization microscope.

Using the nesting of the sets $B_{w}^{n}$ we can make the following definition.

Definition 1. The tip of a map $F \in W^{s}\left(F_{G J M}\right)$ is the point $\tau=\tau(F)=\bigcap_{n \geq 0} B_{0}^{n}$. 


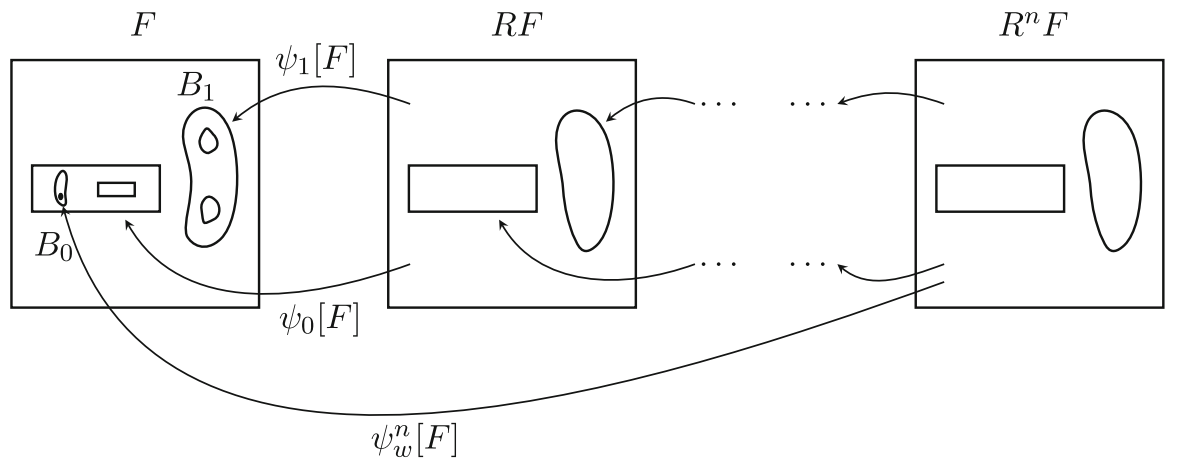

Fig. 1. A schematic picture of the renormalization microscope

In particular the tip $\tau\left(F_{G J M}\right)$ of the renormalization fixed point will be of interest. It can be calculated as the fixed point of $\psi_{0}\left(F_{G J M}\right)$.

$$
\begin{aligned}
\tau\left(F_{G J M}\right) & =\left(x_{\tau}, y_{\tau}\right) \\
& =\psi_{0}\left(\tau\left(F_{G J M}\right)\right) \\
& =\left(\lambda x_{\tau}+p, \mu y_{\tau}\right)
\end{aligned}
$$

from which we can see that $\tau\left(F_{G J M}\right)=\left(\frac{p}{1-\lambda}, 0\right)$. The corresponding point for the EKW renormalization scheme is the origin $(0,0)$ due to the absence of translation by $p$ in $\Lambda$. The nesting also allows us to define the set

$$
\mathcal{O}_{F}=\bigcap_{n=1}^{\infty} \bigcup_{w \in\{0,1\}^{n}} B_{w}^{n-1}
$$

for any $F \in W^{S}\left(F_{G J M}\right)$. In [11] it is proven that the set $\mathcal{O}_{F}$ is an invariant Cantor set on which the dynamics of $F$ is conjugate to the dyadic adding machine. These invariant Cantor sets are the subject of this paper.

The following results will be needed in this paper.

Lemma 2 (Lemma 7.1 of [9]). For every $F \in W(\rho)$, there exists a simply connected closed set $B_{F}$ such that $B_{0}^{1}(F):=\Lambda\left(B_{F}\right) \subset B_{F}$ and $B_{1}^{1}(F):=(F \circ \Lambda)\left(B_{F}\right) \subset B_{F}$ are disjoint, $F\left(B_{1}^{1}(F)\right) \cap B_{0}^{1}(F) \neq \varnothing$ and

$$
\max \left\{\|\mathrm{D} \Lambda\|_{B_{F}},\|\mathrm{D}(F \circ \Lambda)\|_{B_{F}}\right\} \leq \theta:=0.272 .
$$

Note that this lemma is formulated for the EKW renormalization scheme. Here, $W(\rho)$ is the set of infinitely renormalizable area-preserving maps in a ball of radius $\rho$ around an approximation of the fixed point for the EKW renormalization. A similar result is also valid for the renormalization scheme used in $R_{G J M}$ with a slightly better bound, see Proposition 2.3 and Lemma 3.2 of [11]. However since the rescalings for the EKW renormalization scheme differ from the renormalization scheme used in [11] by a translation only depending on $F$ the same bound on the differential is also valid in that setting.

Next lemma states that the rescalings $\psi_{i}[F]$ converge uniformly to the rescalings of the fixed point $F_{G J M}$ under iteration of the renormalization operator $R_{G J M}$. 
Lemma 3 (Lemma 3.1 of [11]). For every $F \in W^{s}\left(F_{G J M}\right)$ and $i \in\{0,1\}$

$$
\lim _{n \rightarrow \infty}\left\|\psi_{i}\left[R_{G J M}^{n-1} F\right]-\psi_{i}\left[F_{G J M}\right]\right\|_{C^{2}}=0 .
$$

Lastly we will also need the rigidity result from [11].

Theorem 4 (Theorem 4.1 of [11]). The Cantor set $\mathcal{O}_{F}$, with $F \in W_{\mathrm{loc}}^{s}\left(F_{G J M}\right)$, is rigid. Namely, there exists an $\alpha_{0}>0$ such that there is a $C^{1+\alpha}$ conjugation $h: \mathcal{O}_{F} \rightarrow \mathcal{O}_{G J M}$ for every $0<\alpha<\alpha_{0}$.

The constant $\alpha_{0}$ appearing in Theorem 4 satisfies $\alpha_{0}>0.237$, see [11].

Remark 5. Note that these three results ultimately depend on computer assistance. Though none of the results of [11] use computer assistance they rely on previous results which do use computer assistance.

We will also need a property of twist maps called the ratchet phenomenon. It says that for a twist map satisfying

$$
\frac{\partial X}{\partial y}>a>0
$$

there are horizontal cones $\Theta_{h}$ and vertical cones $\Theta_{v}$ such that if $p^{\prime} \in p+\Theta_{v}$ then $F\left(p^{\prime}\right) \in F(p)+\Theta_{h}$ and that the angle of the cones depend only on $a$, see e.g. Lemma 12.1 of [14]. The same is true for negative twist maps with

$$
\frac{\partial X}{\partial y}<a<0 .
$$

This condition is clearly satisfied on $B_{F}$ for all maps $F$ considered here since it is compact. We will consider these cones as constant cone fields $\Theta_{h}(p)=\Theta_{h}$ and $\Theta_{v}(p)=$ $\Theta_{v}$ so that, at every point $p, \Theta_{h}(p)$ and $\Theta_{v}(p)$ are just copies of $\Theta_{h}$ and $\Theta_{v}$ in the tangent space at $p$. Using these cone fields we see that the ratchet phenomenon also implies that the differential of $F$ maps the vertical cone field into the horizontal cone field in the corresponding tangent spaces. Thus

$$
\mathrm{D}_{p} F\left(\Theta_{v}(p)\right) \subset \Theta_{h}(F(p)) .
$$

More precisely a positive twist map maps the upper half cone $\Theta_{v}^{+}(p)$ into the right half cone $\Theta_{h}^{+}(F(p))$ and the lower half cone $\Theta_{v}^{-}(p)$ into the left half cone $\Theta_{h}^{-}(F(p))$. A negative twist map changes the signs, i.e. $\Theta_{v}^{+}(p)$ is mapped into $\Theta_{h}^{-}(F(p))$ and $\Theta_{v}^{-}(p)$ is mapped into $\Theta_{h}^{+}(F(p))$.

The results about the structure of the invariant Cantor sets of infinitely renormalizable area-preserving maps proven in this paper are the following two theorems.

Theorem 6. Every map $F \in W^{S}\left(F_{G J M}\right)$ admits a Lipschitz curve containing its invariant Cantor set $\mathcal{O}_{F}$. Furthermore, such a curve can be chosen without self intersections.

Theorem 7. There is no smooth curve containing $\mathcal{O}_{F}$ for any $F \in W_{l o c}^{s}\left(F_{G J M}\right)$.

These results extend those of [3] about nonexistence of smooth curves containing the invariant Cantor set for infinitely renormalizable dissipative maps to the area-preserving setting. 


\section{Existence of Lipschitz Curves}

In this section we prove Theorem 6. The idea is to create a sequence of piecewise smooth curves with uniformly bounded Lipschitz constants that approach the invariant Cantor set. It then follows by the Arzelà -Ascoli theorem that there is a convergent subsequence. The limit of this subsequence is then our sought after Lipschitz curve. The sequence of curves is created inductively by choosing an initial curve $\gamma_{0}$, projecting the previous curve using $\psi_{0}$ and $\psi_{1}$ and then connecting the pieces while at the same time controlling the Lipschitz constants.

Note that the proofs do not use the fact that the maps considered are exact symplectic twist maps. Rather the important part is that the renormalization microscope has a strong enough contraction to compensate for the reparametrization, allowing us to define the sequence of Lipschitz curves for the renormalization fixed point. The uniform convergence of the rescalings to the iterated function system of the renormalization fixed point then allows us to extend this result to all infinitely renormalizable maps. Thus a similar proof would also apply to other renormalization schemes or where a similar iterated function system appears, as long as we have appropriate bounds and convergence.

We begin by showing a bound on the Lipschitz constants on each of the projected pieces.

Lemma 8. Let $F \in W_{\text {loc }}^{s}\left(F_{G J M}\right)$ and let $\gamma:[0,1] \rightarrow B_{F}$ be a piecewise smooth curve with Lipschitz constant $L$. Then for any $n \geq 1$ and any $w \in\{0,1\}^{n}$ the curve $\psi_{w}^{n} \circ \gamma$ has Lipschitz constant at most $\theta^{n} L$.

Proof. Let $s_{1}, s_{2} \in[0,1]$. Then using Lemma 2 we get

$$
\begin{aligned}
\left|\psi_{w}^{n}\left(\gamma\left(s_{1}\right)\right)-\psi_{w}^{n}\left(\gamma\left(s_{2}\right)\right)\right| & \leq \int_{s_{2}}^{s_{1}}\left|\left(\psi_{w}^{n}(\gamma(t))\right)^{\prime}\right| d t \\
& =\int_{s_{2}}^{s_{1}}\left|\mathrm{D} \psi_{w}^{n}(\gamma(t)) \gamma^{\prime}(t)\right| d t \\
& \leq \theta^{n} \int_{s_{2}}^{s_{1}}\left|\gamma^{\prime}(t)\right| d t \\
& \leq \theta^{n} L\left|s_{1}-s_{2}\right| .
\end{aligned}
$$

We are now ready to prove the existence of the Lipschitz curve for the renormalization fixed point $F_{G J M}$. As explained earlier we will achieve the result for all infinitely renormalizable maps as a corollary using the uniform convergence of $\psi_{i}^{n}(F) \rightarrow \psi_{i}\left(F_{G J M}\right)$ from Lemma 3.

Theorem 9. The Eckmann-Koch-Wittwer renormalization fixed point $F_{G J M}$ admits a Lipschitz curve containing its invariant Cantor set $\mathcal{O}_{F_{G J}}$. Furthermore, such a curve can be chosen without self intersections.

Proof. Let $\gamma_{0}:[0,1] \rightarrow B_{F_{G J M}}$ be a piecewise smooth curve without self intersections. We will then construct a new curve $\gamma_{1}$ in the following way: For each $w \in\{0,1\}$ consider the curve $\psi_{w} \circ \gamma_{0}$. Connect $\gamma_{0}(0)$ to $\psi_{0}\left(\gamma_{0}(0)\right), \psi_{0}\left(\gamma_{0}(1)\right)$ to $\psi_{1}\left(\gamma_{0}(0)\right)$ and $\psi_{1}\left(\gamma_{0}(1)\right)$ to $\gamma_{0}(1)$ smooth curves without self intersections and not intersecting either $\psi_{w} \circ \gamma_{0}$. This gives us a piecewise smooth curve $\gamma:[0,5] \rightarrow B_{F_{G J M}}$. On each piece of $\gamma$ corresponding to a piece $\psi_{w} \circ \gamma_{0}$ we affinely reparametrize the curve on an interval 


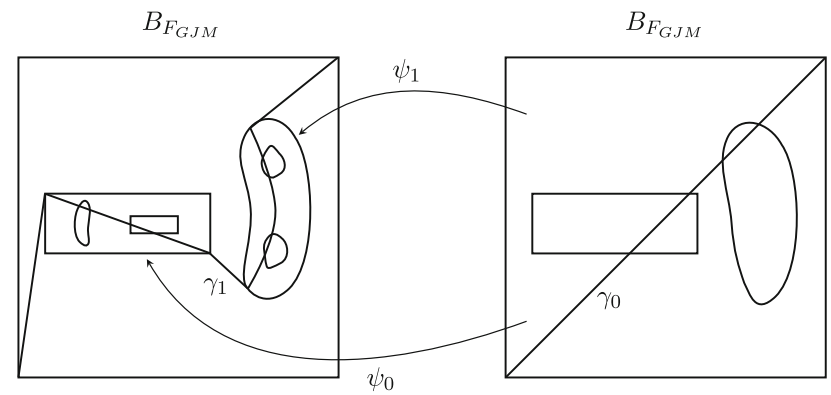

Fig. 2. Constructing $\gamma_{1}$ starting with $\gamma_{0}$ diagonal

of size $\theta$, where $\theta=0.272$ is the constant from Lemma 2. The remaining parts of the interval $[0,1]$ we use to affinely reparametrize the 3 connecting pieces on intervals of size $\frac{1-2 \theta}{3}$. This gives us a piecewise smooth curve $\gamma_{1}$ with Lipschitz constant $L_{1}$ given by the maximum of $\gamma_{1}^{\prime}(t)$ over each smooth piece.

Continuing by induction, assume we have constructed a piecewise smooth curve $\gamma_{k}:[0,1] \rightarrow B_{F_{G J M}}$ and construct a curve $\gamma_{k+1}$ using the same construction as for $\gamma_{1}$ using the same connecting curves. We would now like to estimate the Lipschitz constant of $\gamma_{k+1}$. First let $w \in\{0,1\}$ and consider the piece of the curve $\gamma_{k+1}$ given by $\psi_{w} \circ \gamma_{k} \circ \varphi_{w}$ where $\varphi_{w}$ is the corresponding affine reparametrization. Using Lemma 8 we get

$$
\begin{aligned}
\left|\gamma_{k+1}\left(s_{1}\right)-\gamma_{k+1}\left(s_{2}\right)\right| & =\left|\psi_{w}\left(\gamma_{k}\left(\varphi_{w}\left(s_{1}\right)\right)\right)-\psi_{w}\left(\gamma_{k}\left(\varphi_{w}\left(s_{2}\right)\right)\right)\right| \\
& \leq \theta L_{k}\left|\varphi_{w}\left(s_{1}\right)-\varphi_{w}\left(s_{2}\right)\right| \\
& =\frac{\theta L_{k}}{\theta}\left|s_{1}-s_{2}\right| \\
& =L_{k}\left|s_{1}-s_{2}\right| .
\end{aligned}
$$

Hence on each part $\psi_{w} \circ \gamma_{k} \circ \varphi_{w}$ the curve $\gamma_{k+1}$ has Lipschitz constant at most $L_{k}$. Outside of all $B_{w}^{1}\left(F_{G J M}\right)$ the curve $\gamma_{k+1}$ looks exactly like $\gamma_{1}$ and hence the Lipschitz constant here is at most $L_{1}$. Since the concatenation of two Lipschitz curves is again a Lipschitz curve with Lipschitz constant equal to the larger of the original Lipschitz constants we then have $L_{k+1} \leq \max \left(L_{k}, L_{1}\right) \leq \max \left(L_{k-1}, L_{1}\right) \leq \cdots \leq L_{1}$ since $\gamma_{k+1}$ is the concatenation of five Lipschitz curves, each with Lipschitz constant $L_{k}$ or $L_{1}$.

We then have a sequence of Lipschitz curves $\gamma_{k}$ with Lipschitz constants uniformly bounded by $L_{1}$. By the Arzelà-Ascoli theorem there is then a subsequence $\gamma_{k_{l}}$ converging uniformly to a Lipschitz curve $\gamma$. The image of this curve $\gamma$ must then contain the invariant Cantor set $\mathcal{O}_{F_{G J M}}$ of $F_{G J M}$ since it intersects $B_{w}^{n}$ for every $w \in\{0,1\}^{n}$ and every $n$.

Furthermore, by choosing the original curve $\gamma_{0}$ and the connecting curves appropriately the final curve will have no self intersections. Note that the maps $\psi_{w}$ are diffeomorphisms and hence will never introduce self intersections, so the only source of self intersections are the connecting curves.

Corollary 10. Every map $F \in W^{s}\left(F_{G J M}\right)$ admits a Lipschitz curve containing its invariant Cantor set $\mathcal{O}_{F}$. Furthermore, such a curve can be chosen without self intersections. 
Proof. By the uniform convergence of $\psi_{i}\left[R_{G J M}^{n-1} F\right] \rightarrow \psi_{i}\left[F_{G J M}\right]$ given by Lemma 3 we can find an $N$, depending only on $F$, large enough so that for every $n \geq N$ Lemma 2 is true for each $\psi_{i}\left[R_{G J M}^{n} F\right]$, possibly with a slightly larger $\theta$. Thus the construction from Theorem 9 over each $B_{w}\left(R^{n} F\right)$ will not increase the Lipschitz constant for $n \geq N$. Similarly the curves connecting the pieces $B_{w}\left(R^{n} F\right)$ will also be $C^{1}$-close to the connecting curves for the fixed point $F_{G J M}$ case so the Lipschitz constant will be uniformly bounded outside $B_{w}\left(R^{n} F\right)$ as well. Hence we again get a sequence of Lipschitz curves $\gamma_{k}$ with uniformly bounded Lipschitz constants $L_{k}$. By applying the Arzelà -Ascoli theorem we now get a Lipschitz curve containing the invariant Cantor set $\mathcal{O}_{F}$.

Again, choosing the initial curve and the connecting curves appropriately we can get a curve with no self intersections.

Note that since the curve $\gamma$ constructed above is Lipschitz it is in particular also rectifiable. In this sense we have proven the corresponding result of [3] for infinitely renormalizable area-preserving maps.

\section{Nonexistence of Smooth Curves}

We will now prove Theorem 7. We will first consider only the renormalization fixed point $F_{G J M}$. In order to show that there is no smooth curve containing $\mathcal{O}_{F_{G J M}}$ we will first show that $\mathcal{O}_{F_{G J M}}$ does not admit a continuous field of directions. Following the ideas of [3] this will then imply that $\mathcal{O}_{F_{G J M}}$ does not admit a smooth curve containing it either. Using rigidity, the result for any $F \in W^{s}\left(F_{G J M}\right)$ is then a simple corollary.

As opposed to the proof for Lipschitz curves this proof does use the fact that the fixed point is a twist map in an essential way.

We begin with a lemma about the EKW renormalization fixed point.

Lemma 11. At the tip $\tau=\bigcap_{n \geq 0} B_{0}^{n}\left(F_{E K W}\right)=(0,0)$ of the EKW renormalization fixed point $F_{E K W}$ we have

$$
\frac{\partial X}{\partial x}(\tau)<0
$$

Proof. Note that the point $(X, x)=(1,0)$ corresponds to the tip $\tau$ of $F_{E K W}$ by the normalization condition $s(1,0)=0$.

By the normalization condition $s_{1}(1,0)=1$ it follows that

$$
\frac{\partial X}{\partial y}(0,0)=-\frac{1}{s_{1}(1,0)}=-1
$$

so that the map $F_{E K W}$ is in fact a negative twist map and we must have that $s_{1}(X, x)>0$ everywhere. Since

$$
\frac{\partial X}{\partial x}(\tau)=-\frac{s_{2}(1,0)}{s_{1}(1,0)}
$$

we thus need to show that $s_{2}(1,0)>0$.

Consider first the equation for the renormalization fixed point for $s$,

$$
s(x, X)=\frac{1}{\mu} s(z(x, X), \lambda X) .
$$


Differentiating with respect to $X$ gives us

$$
s_{2}(x, X)=\frac{1}{\mu}\left(s_{1}(z(x, X), \lambda X) z_{2}(x, X)+\lambda s_{2}(z(x, X), \lambda X)\right) .
$$

Solving for $s_{2}$, evaluating at the point $(1,0)$ and using the normalization condition $z(1,0)=1$ we get

$$
s_{2}(1,0)=\frac{z_{2}(1,0)}{\mu-\lambda},
$$

therefore showing that $s_{2}(1,0)>0$ is reduced to showing that $z_{2}(1,0)>0$.

To do this we first differentiate Eq. 2.6 with respect to $y$ and get

$$
s_{2}(\lambda x, z(x, X)) z_{2}(x, X)+\lambda s_{1}(\lambda X, z(x, X))+s_{2}(\lambda X, z(x, X)) z_{2}(x, X)=0 .
$$

Evaluating at $(x, X)=(1,0)$ yields

$$
\begin{aligned}
& s_{2}(\lambda, 1) z_{2}(1,0)+\lambda s_{1}(0,1)+s_{2}(0,1) z_{2}(1,0)=0 \\
\Rightarrow & z_{2}(1,0)=-\lambda \frac{s_{1}(0,1)}{s_{2}(\lambda, 1)+s_{2}(0,1)} .
\end{aligned}
$$

Since $-\lambda s_{1}(0,1)>0$ due to the negative twist condition and $\lambda<0$ we must then show that $s_{2}(\lambda, 1)+s_{2}(0,1)$ is also positive. To do this we differentiate Equation 2.6 with respect to $x$ and get

$$
\lambda s_{1}(\lambda x, z(x, X))+s_{2}(\lambda x, z(x, X)) z_{1}(x, X)+s_{2}(\lambda X, z(x, X)) z_{1}(x, X)=0 .
$$

Evaluating at the point $(x, X)=(1,0)$ then gives us

$$
\begin{aligned}
& \lambda s_{1}(\lambda, 1)+\mu s_{2}(\lambda, 1)+\mu s_{2}(0,1)=0 \\
\Rightarrow & s_{2}(\lambda, 1)+s_{2}(0,1)=-\frac{\lambda}{\mu} s_{1}(\lambda, 1)
\end{aligned}
$$

which is positive by the negative twist condition forcing $s_{1}>0$ everywhere. Thus $z_{2}(1,0)$ is positive and hence also $s_{2}(1,0)$ is positive, meaning that

$$
\frac{\partial X}{\partial x}(\tau)=-\frac{s_{2}(1,0)}{s_{1}(1,0)}<0 .
$$

We will now connect $F_{E K W}$ with $F_{G J M}$ by another lemma.

Lemma 12. The maps $F_{E K W}$ and $F_{G J M}$ are conjugate by a translation in the $x$ direction.

Proof. Recall that the renormalization operator $R_{E K W}$ is defined by

$$
R_{E K W}(F)=\Lambda^{-1} \circ F^{2} \circ \Lambda
$$


where $\Lambda(x, y)=(\lambda x, \mu y)$. We begin by noticing that $\psi_{0}=h^{-1} \circ \Lambda \circ h$ where $h$ is the translation in the $x$-direction by the tip $\tau=\frac{p_{F}}{1-\lambda}$, i.e. $h(x, y)=\left(x-\frac{p}{1-\lambda}, y\right)$. We then get:

$$
\begin{aligned}
h^{-1} \circ F_{E K W} \circ h & =h^{-1} \circ R_{E K W}\left(F_{E K W}\right) \circ h \\
& =h^{-1} \circ \Lambda^{-1} \circ F_{E K W}^{2} \circ \Lambda \circ h \\
& =\left(h^{-1} \circ \Lambda^{-1} \circ h\right) \circ\left(h^{-1} \circ F_{E K W}^{2} \circ h\right) \circ\left(h^{-1} \circ \Lambda \circ h\right) \\
& =\psi_{0}^{-1} \circ\left(h^{-1} \circ F_{E K W}^{2} \circ h\right) \circ \psi_{0} \\
& =R_{G J M}\left(h^{-1} \circ F_{E K W} \circ h\right)
\end{aligned}
$$

so $F_{G J M}=h^{-1} \circ F_{E K W} \circ h$ is a fixed point of $R_{G J M}$.

Since such a conjugacy does not change the values of derivatives, $F_{G J M}$ must also satisfy

$$
\frac{\partial X}{\partial x}<0
$$

at the tip $\tau=\left(\frac{p}{1-\lambda}, 0\right)$ of $F_{G J M}$. Note also that this coordinate change corresponds to the generator $\sigma_{-1,0}^{1}$ from [10], which in turn corresponds to the eigenvalue $\lambda^{-1}$ of $\mathcal{D} R$ at the fixed point. With this we can now prove that the invariant Cantor set $\mathcal{O}_{F_{G J}}$ of the renormalization fixed point $F_{G J M}$ does not admit a continuous invariant direction field.

Theorem 13. There is no continuous invariant direction field on the invariant Cantor set $\mathcal{O}_{F_{G J M}}$ of $F_{G J M}$.

The basic idea of the proof is to assume to the contrary that there is such a continuous invariant direction field and show that it must then contain directions on either side of the vertical line. Using $\psi_{0}$ we can project these towards the tip of $F_{G J M}$ at which point these directions become horizontal in opposite directions, contradicting continuity of the field of directions.

Proof. Assume, towards a contradiction, that there is a continuous invariant line field $\ell$ on $\mathcal{O}_{F_{G J M}}$. Let $\Theta_{v}^{ \pm}(p)$ and $\Theta_{h}^{ \pm}(p)$ be the vertical and horizontal cones at $p \in \mathcal{O}_{F_{G J M}}$ from the ratchet phenomenon. We then know, since $F_{G J M}$ is a negative twist map, that $\mathrm{D} F_{G J M}\left(\Theta_{v}^{+}(p)\right) \subset \Theta_{h}^{-}\left(F_{G J M}(p)\right)$ and $\mathrm{D} F_{G J M}\left(\Theta_{v}^{-}(p)\right) \subset \Theta_{h}^{+}\left(F_{G J M}(p)\right)$. By reversibility, $F^{-1}=T \circ F \circ T$, we also have that $\mathrm{D} F_{G J M}^{-1}\left(\Theta_{v}^{+}(p)\right) \subset \Theta_{h}^{+}\left(F_{G J M}^{-1}(p)\right)$ is the reflection in the horizontal axis of $\mathrm{D} F_{G J M}\left(\Theta_{v}^{-}(T(p))\right)$ since

$$
\mathrm{D} F_{G J M}^{-1}\left(\Theta_{v}^{+}(p)\right)=\mathrm{D}\left(T \circ F_{G J M} \circ T\right)\left(\Theta_{v}^{-}(T(p))\right)
$$

and in the same way $\mathrm{D} F_{G J M}^{-1}\left(\Theta_{v}^{-}(p)\right) \subset \Theta_{h}^{-}\left(F_{G J M}^{-1}(p)\right)$ is the reflection in the horizontal axis of $\mathrm{D} F_{G J M}\left(\Theta_{v}^{+}(T(p))\right)$.

Using this fact, suppose first that $\ell(\tau)$ is not horizontal. Since $\left.F_{G J M}^{2^{n}}\right|_{B_{0}^{n}}=\psi_{0}^{n} \circ$ $\left.F_{G J M} \circ \psi_{0}^{-n}\right|_{B_{0}^{n}}$ we can find $N$ large enough so that for every $n \geq N$ we have $\mathrm{D}_{\tau}\left(\psi_{0}^{-n}\right)(\ell(\tau)) \in \Theta_{v}^{ \pm}(\tau)$. Applying $\mathrm{D} F_{G J M}$ and $\mathrm{D} F_{G J M}^{-1}$ the resulting directions must then be on opposite sides of the vertical. Finally, projecting back to $B_{0}^{n}$ with $\psi_{0}^{n}$ these 
directions approach the horizontal on opposite sides of the vertical axis. Since $\ell$ is invariant under $F_{G J M}$ it must also be invariant under $F_{G J M}^{2^{n}}$. Thus $\ell\left(F_{G J M}^{2^{n}}(\tau)\right)$ and $\ell\left(F_{G J M}^{-2^{n}}(\tau)\right)$ are close to the horizontal axis on opposite sides of the vertical axis. Since furthermore we can choose $n$ large enough so that $F_{G J M}^{2^{n}}(\tau)$ and $F_{G J M}^{-2^{n}}(\tau)$ are as close to $\tau$ as we want this contradicts continuity of $\ell$.

Next suppose that $\ell(\tau)$ is horizontal. By Lemma 11 we have that $\ell\left(F_{G J M}(\tau)\right)$ must then be on the opposite side of the vertical axis. Using the same argument as in the previous case we can then find points arbitrarily close to $\tau$ where $\ell$ is on the other side of the vertical axis, again contradicting continuity.

We conclude that there can be no continuous invariant direction field.

Following [3] we now prove that this also implies that there is no continuous invariant line field on $\mathcal{O}_{F_{G J M}}$.

Lemma 14. The invariant Cantor set $\mathcal{O}_{F_{G J}}$ does not admit a continuous invariant line field.

Proof. Suppose towards a contradiction that there is a continuous invariant line field on $\mathcal{O}_{F_{G J}}$. By compactness we can find an $n_{0}$ large enough so that the part of the line field in $\mathcal{O}_{F_{G J M}} \cap B_{w}^{n_{0}}$ can be continuously oriented for every $w \in\{0,1\}^{n_{0}}$. Furthermore, since the pieces are all disjoint, choosing a continuous orientation on each of them gives us a continuous orientation of the line field on the entire set $\mathcal{O}_{F_{G J M}}$. This gives us a continuous direction field on $\mathcal{O}_{F_{G J M}}$ and it allows us to partition $\mathcal{O}_{F_{G J M}}$ into two parts: $\mathcal{O}_{F_{G J M}}^{+}$on which $F_{G J M}$ preserves the orientation and $\mathcal{O}_{F_{G J M}}^{-}$on which $F_{G J M}$ reverses the orientation. By continuity both of these sets must be both closed and open. Then for every large enough $n$ every $\mathcal{O}_{F_{G J M}} \cap B_{w}^{n}$ is entirely contained in either $\mathcal{O}_{F_{G J M}}^{+}$or $\mathcal{O}_{F_{G J M}}^{-}$. Hence $F_{G J M}^{2^{n}} \mid \mathcal{O}_{F_{G J M}} \cap B_{0}^{n}$ either preserves or reverses orientation and therefore $\left.F_{G J M}^{2^{n+1}}\right|_{\mathcal{O}_{F_{G J M}} \cap B_{0}^{n+1}}$ must preserve orientation. Using the fact that $F_{G J M}$ is the renormalization fixed point this direction field can then be pulled back to a new continuous invariant direction field on $\mathcal{O}_{F_{G J M}}$, but this contradicts Theorem 13. Therefore $\mathcal{O}_{F_{G J M}}$ cannot admit a continuous invariant line field.

Finally, using the same idea as in [3], we can now prove that there is no smooth curve containing $\mathcal{O}_{F_{G J M}}$.

Theorem 15. There is no smooth curve containing $\mathcal{O}_{F_{G J M}}$.

Proof. Assume that there is a smooth curve $\gamma$ containing $\mathcal{O}_{F_{G J M}}$. Then the line field $\ell$ of tangent lines to $\gamma$ gives us a continuous line field on $\mathcal{O}_{F_{G J M}}$. Furthermore it is invariant since it satisfies

$$
\ell(p)=\lim _{q \rightarrow p} l(p, q)
$$

where $l(p, q)$ is the line passing through $p, q \in \mathcal{O}_{F_{G J}}$. Using Lemma 14 this contradicts Theorem 13.

Using Theorem 4 we get the following corollary of Theorem 15.

Corollary 16. There is no smooth curve containing $\mathcal{O}_{F}$ for any $F \in W_{l o c}^{s}\left(F_{G J M}\right)$. 
Acknowledgements. The author would like to thank Professor Marco Martens for his invaluable guidance and enlightening discussions during the author's stay at Stony Brook University as a visiting research scholar during the spring of 2016, during which time most of this work was done. The author's visit to Stony Brook University was supported by the Swedish Foundation for international Cooperation in Research and Higher Education (STINT) grant number 2012-2153.

Open Access This article is distributed under the terms of the Creative Commons Attribution 4.0 International License (http://creativecommons.org/licenses/by/4.0/), which permits unrestricted use, distribution, and reproduction in any medium, provided you give appropriate credit to the original author(s) and the source, provide a link to the Creative Commons license, and indicate if changes were made.

\section{References}

1. Avila, A., Lyubich, M.: The full renormalization horseshoe for unimodal maps of higher degree: exponential contraction along hybrid classes. Publ. Math. Inst. Hautes Études Sci. 114, 171-223 (2011)

2. Collet, P., Eckmann, J.-P., Koch, H.: Period doubling bifurcations for families of maps on $\mathbf{R}^{n}$. J. Stat. Phys. 25(1), 1-14 (1981)

3. De Carvalho, A., Lyubich, M., Martens, M.: Renormalization in the Hénon family. I. Universality but non-rigidity. J. Stat. Phys. 121(5-6), 611-669 (2005)

4. de Faria, E., de Melo, W., Pinto, A.: Global hyperbolicity of renormalization for $C^{r}$ unimodal mappings. Ann. Math. (2) 164(3), 731-824 (2006)

5. Eckmann, J.-P., Koch, H., Wittwer, P.: A computer-assisted proof of universality for area-preserving maps. Mem. Am. Math. Soc. 47(289), vi+122 (1984)

6. Feigenbaum, M.J.: Quantitative universality for a class of nonlinear transformations. J. Stat. Phys. 19(1), 25-52 (1978)

7. Feigenbaum, M.J.: The universal metric properties of nonlinear transformations. J. Stat. Phys. 21(6), 669706 (1979)

8. Gaidashev, D., Johnson, T.: Dynamics of the universal area-preserving map associated with period doubling: hyperbolic sets. Nonlinearity 22(10), 2487 (2009)

9. Gaidashev, D., Johnson, T.: Dynamics of the universal area-preserving map associated with perioddoubling: stable sets. J. Mod. Dyn. 3(4), 555-587 (2009)

10. Gaidashev, D., Johnson, T.: Spectral properties of renormalization for area-preserving maps. Discrete Contin. Dyn. Syst. 36(7), 3651-3675 (2016)

11. Gaidashev, D., Johnson, T., Martens, M.: Rigidity for infinitely renormalizable area-preserving maps. Duke Math. J. 165(1), 129-159 (2016)

12. Gaidashev, D., Yampolsky, M.: Golden mean Siegel disk universality and renormalization. ArXiv e-prints (2016)

13. Gambaudo, J.-M., van Strien, S., Tresser, C.: Hénon-like maps with strange attractors: there exist $C^{\infty}$ Kupka-Smale diffeomorphisms on $S^{2}$ with neither sinks nor sources. Nonlinearity 2(2), 287-304 (1989)

14. Golé, C.: Symplectic Twist Maps, Volume 18 of Advanced Series in Nonlinear Dynamics. Global variational techniques, World Scientific Publishing Co., Inc., River Edge (2001)

15. Lyubich, M.: Feigenbaum-Coullet-Tresser universality and Milnor's hairiness conjecture. Ann. Math. (2) 149(2), 319-420 (1999)

16. Lyubich, M., Martens, M.: Probabilistic Universality in Two-dimensional Dynamics. ArXiv e-prints (2011)

17. Lyubich, M., Martens, M.: Renormalization of Hénon maps. In: Dynamics, Games and Science. I, Volume 1 of Springer Proc. Math., pp. 597-618. Springer, Heidelberg (2011)

18. Martens, M.: The periodic points of renormalization. Ann. Math. (2) 147(3), 543-584 (1998)

19. Sullivan, D.: Bounds, quadratic differentials, and renormalization conjectures. In American Mathematical Society centennial publications, Vol. II (Providence, RI, 1988), pp. 417-466. American Mathematical Society, Providence (1992)

20. Tresser, C., Coullet, P.: Itérations d'endomorphismes et groupe de renormalisation. C. R. Acad. Sci. Paris Sér. A B 287(7), A577-A580 (1978)

21. Yampolsky, M.: The attractor of renormalization and rigidity of towers of critical circle maps. Commun. Math. Phys. 218(3), 537-568 (2001)

22. Yampolsky, M.: Hyperbolicity of renormalization of critical circle maps. Publ. Math. Inst. Hautes Études Sci. 96, 1-41 (2002, 2003)

Communicated by C. Liverani 